\title{
Gain/Loss Periodic Spatial Modulated Materials on a Wavelength Scale
}

\author{
Kestutis Staliunas ${ }^{1}$, Ramon Herrero ${ }^{2}$, Ramon Vilaseca ${ }^{2}$ \\ 1. ICREA \& Dep. de Física, Universitat Politècnica de Catalunya, Colom 11, 08222 Terrassa, Spain \\ 2. Dep. de Física, Universitat Politècnica de Catalunya, Colom 11, 08222 Terrassa, Spain \\ e-mail: kestutis.staliunas@icrea.es
}

\begin{abstract}
We predict self-collimation and superdiffusion of light propagating in materials with periodically modulated gain/loss profile. The modulation is parallel and transverse to the light propagation direction and its periodicity is on the wavelength scale. The self-collimation (or diffraction-free propagation) of the beams is found, which is similar to that observed in photonic crystals. The superdiffusion is also predicted, which means a dependence of the beam amplification on the propagation direction, which results in the spatial frequency filtering of the beams. We justify the predictions by the numerical simulations of the paraxial wave propagation equations.
\end{abstract}

Keywords: Photonic crystals, modulated materials, micro and nanophotonics.

\section{INTRODUCTION}

It is well known, that the materials with the refractive index modulated in space on the wavelength scale, i.e. the so-called photonic crystals (PCs), bring about a significant modification of the propagation properties of waves, both in time and space domains. In time domain the usual (temporal) dispersion is modified, and photonic bandgaps appear in the frequency spectra [1]. The photonic bandgaps are, perhaps, the most celebrated property of photonic crystals. In the space domain, the spatial dispersion (diffraction) can also be modified. This leads, analogously to the temporal case, to the appearance of the bandgaps in terms of the propagation direction (i.e. in the propagation wavevector domain). The character of diffraction within the propagation bands can be also modified: it can result in the negative diffraction leading to lensing and superlensing [2], it can also result to the zero-diffraction leading to the self-collimation [3] (also called diffractionless or subdiffractive propagation $[4,5])$.

Whereas the PCs, the materials with spatially modulated refractive index, are the subject of intensive study, the seemingly analogous materials, those with gain/loss modulation (GLM), do not enjoy a sensible interest. One reason for such a "discrimination" is that the GLM materials do not possess the celebrated property of the PCs the bandgaps. If the refractive index modulation pushes the frequencies of the harmonic field components one from another, and thus opens the bandgaps around their cross-points, the modulation of the gain/loss does the opposite - it closes the bandgaps. In this way, the bandgaps do not appear in the GLM systems. We show, however, that the modification of the spatial dispersion in GLM systems leads to a variety of nontrivial spatial effects. Indeed the spatial propagation effects in the PCs are due to the deformation (flattening) of the interacting (mutually pushing) spatial dispersion curves. In the present case of GLM, the spatial dispersion curves also become substantially modified due to the mode pulling and locking. The novel spatial phenomena associated with these deformations of the spatial dispersion curves is the topic of the present paper.

\section{THE MODEL}

In analogy with the previous studies of the light propagation in PCs [5], we use the paraxial approximation: The gain/loss modulation (GLM) function is $g(x, z)=4 g_{0} \cos \left(q_{\perp} x\right) \cos \left(q_{\|} z\right)$, which represents a spatially-periodic lattice with transverse and longitudinal modulation wavenumbers $\left(q_{\perp}, q_{\|}\right)$. Gain (loss) occurs in the areas $g(x, z)>0(g(x, z)<0)$ thus the average gain is considered to be zero. The normalized paraxial propagation equation reads:

$$
\frac{\partial A}{\partial Z}=\left(i \frac{\partial^{2}}{\partial X^{2}}+V(X, Z)\right) A
$$

where $A(X, Z)$ is the slowly-varying envelope of the electromagnetic field. The normalizations are adapted from [5], where $X=x q_{\perp}$ is the normalized transverse coordinate; $Z=z q_{\perp}^{2} / 2 k_{0}$ is the normalized longitudinal coordinate $\left(k_{0}=2 \pi / \lambda\right.$ is the wavenumber of the incident monochromatic light), and $V(X, Z)=4 m \cdot \cos (X) \cos \left(Q_{\|} Z\right)$, where $Q_{\|}=2 q_{\|} k_{0} / q_{\perp}^{2}$ is the normalized longitudinal component of the wavevector of the modulation, and $m$ is the normalized modulation parameter $m=g_{0} k_{0} / 4 q_{\perp}^{2}$. The modulation is 
considered harmonic, but the analogous results are expected for the arbitrary periodic modulations with the same lattice wavevectors. We note that the average GLM profile in the transverse direction (i.e. integrated over one longitudinal modulation period) is homogeneous, therefore the system remains translational invariant in the transverse space.

For the analytical treatment we introduce a harmonic expansion in terms of the periodicity of the gain/loss profile:

$$
A(X, Z)=\exp \left(i K_{\perp} X\right)\left[a_{0}(Z)+a_{-1}(Z) \exp \left(-i X-i Q_{\|} Z\right)+a_{1}(Z) \exp \left(+i X-i Q_{\|} Z_{\|}\right)+\ldots\right]
$$

Which, inserted into (3), (4), and after truncation to three most relevant harmonics (see Fig. 2b), results into:

$$
\begin{gathered}
d a_{0} / d Z=-i K_{\perp}^{2} a_{0}+m\left(a_{1}+a_{-1}\right) \\
d a_{-1} / d Z=-i\left(K_{\perp}-1\right)^{2} a_{-1}+i Q_{\|} a_{-1}+m a_{0} \\
d a_{1} / d Z=-i\left(K_{\perp}+1\right)^{2} a_{1}+i Q_{\|} a_{1}+m a_{0}
\end{gathered}
$$

A similar equation system was used for the study of the self-collimation in PCs [5], except for the linear coupling coefficients, which are real-valued here in GLM system. We perform next a standard analysis of Eqs. (3) by looking for the exponentially growing eigenmodes: $a_{0}, a_{-1}, a_{1} \propto \exp \left(i K_{\|} z\right)$, where the exponential growth is incorporated into the imaginary part of $K_{\|}$, and present the results in two distinct regimes: self-collimating regime and spatial filtering regime.

\section{SELF COLLIMATION REGIME}

Self-collimation occurs for $Q_{\|}>1$, in a parameter range, which is "above" the triple-cross-point of the dispersion curves of harmonic components. This is in contrast with the PCs case, where the condition $Q_{\|}<1$ holds for the self-collimation regime [5]. By choosing a particular amplitude $m$ for the GLM, one can achieve the flattening of the dispersion curve for a given geometry, as shown in Fig. 1b. The behavior of the imaginary part of the propagation wavenumber of the Bloch mode is shown in Fig. 1c. Whereas the self-collimated modes propagate with a net gain/loss equal to zero (for the zero average gain), the modes propagating at some angles (those around the values corresponding to the crossing of the dispersion curves of two neighboring harmonic components) mutually lock and amplify. This means, that the self-collimated beam develop an instability with respect to the sidebands during the propagation even for the zero average gain.
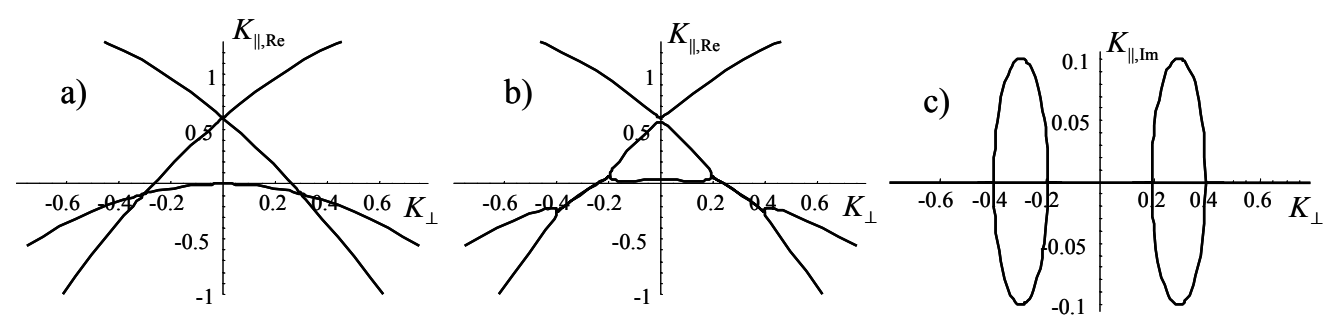

Figure 1. Propagation eigenvalues (real parts of $\left.K_{\|}\right)(a, b)$, and the growth/decay exponents (imaginary parts of $K_{\|}$) (c) for the three-coupled-mode model (6), in the self-collimation regime for $Q_{\|}=1.6$. (a) illustrates the geometry of the dispersion parabolas of harmonic components with vanishing coupling $(m \rightarrow 0)$. (b) shows the development of a self-collimation plateau on the dispersion curves of the Bloch modes for $m=0.1$.

The predicted self-collimation was confirmed by numerically solving the paraxial equation (1) taking into account explicitly the gain/loss modulation. Fig. 2a shows that the narrow beam, upon entering the modulated material propagates without any diffraction. However two sidebands grow, and after some propagation distance they become dominating (note that the transverse distributions of the field are normalized to its peak value at every propagation distance in Fig. 2a). The nondiffractive character of the beam propagation is demonstrated in Fig. 2c, which compares the width of the central, self-collimated, part of the beam with the width of an analogous beam propagating in a homogeneous material. The small oscillations appear because of the influence of the amplifying sidebands. 

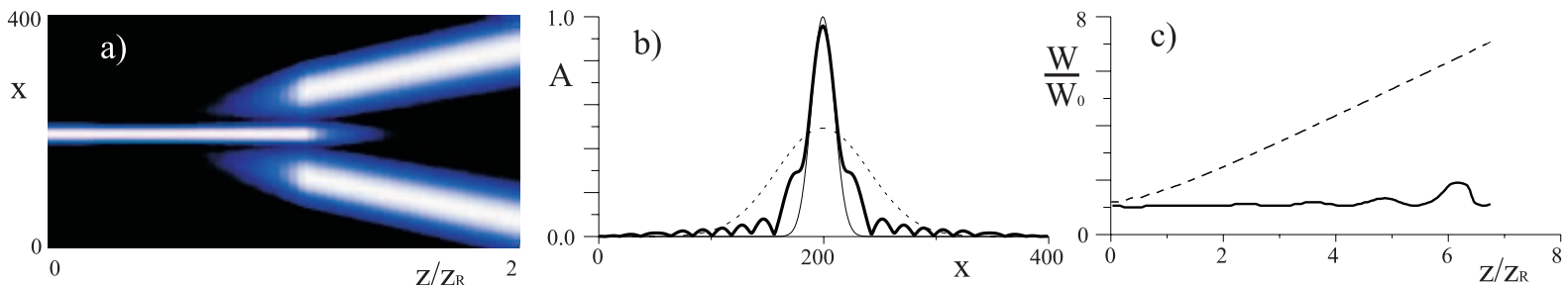

Figure 2. a) Field amplitude for a propagating beam through a GLM material with $m=0.1$ and $Q_{\|}=1.6$. The peak amplitude in each transverse section has been normalized to unity. b) Profiles of the initial Gaussian beam (thin solid curve) and propagated beam a distance $Z=4 Z_{R}$ along the modulated material (bold curve) and along a homogeneous material (dashed curve). c) Width of the central part of the beam propagating through the modulated and a homogeneous material (dashed curve).

\section{SPACE FILTERING REGIME}

Close to the triple-cross-point of the dispersion parabolas, i.e. at around $Q_{\|}=1$ the above described selfcollimation is absent. The geometry of the dispersion curves of the harmonic components is given in Fig. 3a. The main characteristic of this geometry is that for a definite range of amplitudes of the gain/loss modulation (as shown in Fig. $3 \mathrm{~b}$ and Fig. $3 \mathrm{c}$ ) a narrow gain profile along the optical axis appears, which indicates a strong angular dependence of the gain around $K_{\perp}=0$. The latter acts as a spatial filtering, as it results in a discrimination of the high transverse harmonics.
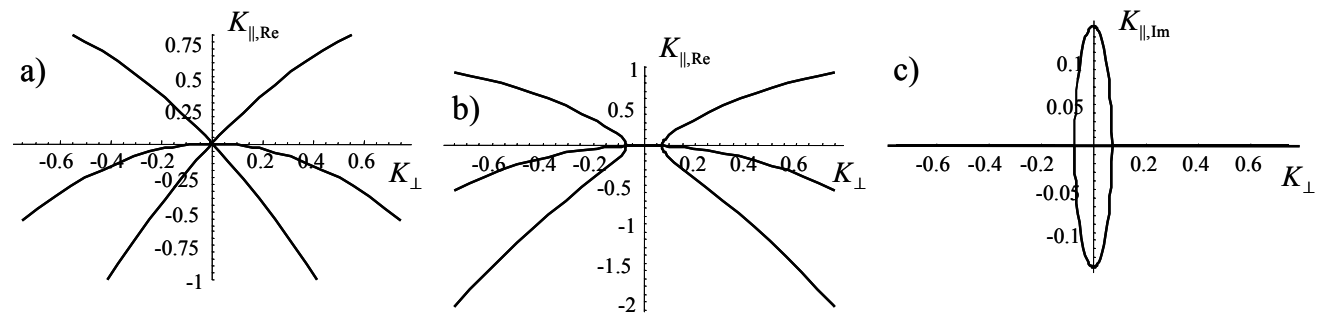

Figure 3. Dispersion and gain profiles for the three-coupled-mode model in the spatial filtering regime with $m=0.1$ and $Q_{\|}=1.0$. a) Geometry of dispersion parabolas of the three harmonic components considered in the model. b) The propagation eigenvalues (real parts of $K_{\|}$) presenting a plateau of mode-locking and c) the growth exponents (imaginary parts of $K_{\|}$) presenting an angular dependent gain profile.

We prove numerically with the paraxial model (1) the spatial filtering effect based on the angular selectivity of the amplification. Fig. 4a shows the evolution of the profile of the amplifying beam, exhibiting its diffusive character of broadening. We note that the diffusive broadening follows a $W \propto Z^{1 / 2}$ dependence in contrast to the diffractively spreading beams obeying $W \propto Z^{1}$. This dependence is shown in Fig. 4b. The angular distribution of the gain is presented in Fig. 5c, obtained by numerical integration of plane waves entering into the media of modulated gain/loss at varying angles. The narrow angular spectrum of amplification corresponds well with that calculated semianalytically in Fig. 3.
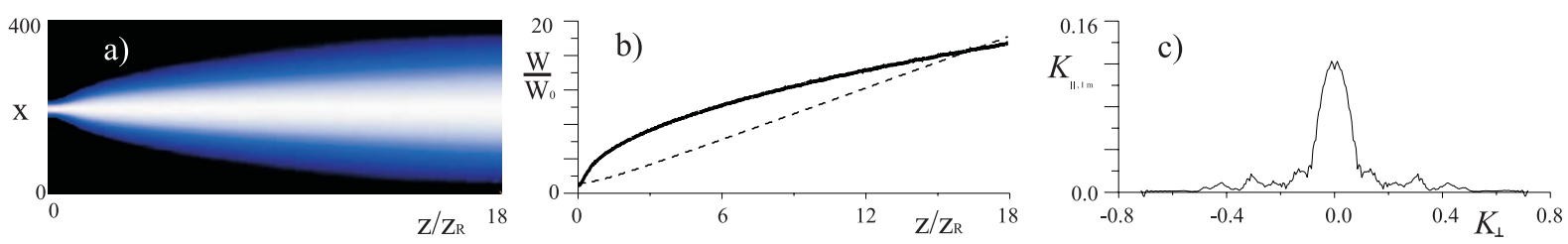

Figure 4. a) Propagation of a Gaussian beam through gain/loss modulated medium with $m=0.1$ and $Q_{\|}=1.0$. The initial beam is as in Fig. 3. b) The width of the beam propagated through the modulated medium (thick line) and homogeneous material (dashed line). c) Imaginary part of the propagation wavenumber obtained from the propagation of plane waves through the material in different directions.

\section{CONCLUSIONS}

Concluding, we have predicted and demonstrated theoretically (by means of semianalytical expansion into three harmonics, and by numerical integration of the paraxial model) the existence of two novel phenomena of the propagation of the beams in media with gain/loss modulation in two-dimensional systems (one longitudinal and one transverse direction). These phenomena are: the self collimation, similar to that predicted in PCs; and the 
spatial filtering, which has no analog in PCs. Seemingly the second phenomenon, spatial filtering, is of a greater technological importance, as it should allow preparing highly directional beams. Self-collimation as shown above, is obscured by the fact that the amplification of the modes at some angles to the optical axis occur, which means the modulation instability of the beam propagating in self-collimated regime.

The presented analysis, which concerns a two dimensional gain/loss modulation, could be also extended into the three-dimensional case. The basic discussion on the crossing and locking of dispersion curves can be extended to the crossing and locking of the dispersion surfaces. The essential difference with the twodimensional case is the symmetry of the lattice in the transverse plane, as explored e.g. in [6] in the case of PCs. It comes out from a preliminary study that the different symmetries of the modulation in the transverse plane result in a different transverse distribution of the amplified wavenumbers in the spatial Fourier domain, e.g. a square lattice results in approximately square distributions, and the lattices of hexagonal or octagonal symmetry result in more round ones.

The present analysis in paraxial approximation should be extended to the full, nonparaxial analysis. It is expected that the predicted phenomena will pertain also in nonparaxial case, due to analogy between the paraxial and nonparaxial self-collimation in PCs [7]. And also the predicted directionality of amplification in the GLM materials can be applied to the active/passive resonators with the GLM material inside, generalising the recently predicted effects in PC filled resonators [8].

\section{ACKNOWLEDGEMENTS}

The work was financially supported by Spanish Ministerio de Educación y Ciencia and European Union FEDER through project FIS2005-07931-C03-03 and FIS2008-06024-C03-02.

\section{REFERENCES}

[1] E. Yablonovitch, Phys. Rev. Lett. 58, 2059 (1987); S. John, Phys. Rev. Lett. 58, 2486 (1987).

[2] H. Haus, and L. Molter-Orr, IEEE J. Quantum Electron. 19, 840 (1983).

[3] H. Kosaka et al., Appl. Phys. Lett. 74, 1212 (1999).

[4] D. N. Chigrin et al., Optics Express, 11, 1203 (2003); R. Illiew et al., Appl. Phys. Lett. 85, 5854 (2004).

[5] K. Staliunas and R. Herrero, Phys. Rev. E 73, 016601 (2006).

[6] K. Staliunas, R. Herrero and G.J. de Valcárcel, Phys. Rev. A 75 011604(R) (2007).

[7] Yu. Loiko, et al., Optics Communications, 269, 128 (2007).

[8] M. Peckus, et al., Phys. Rev. A, 79, 033806 (2009). 